\title{
OPTIMIZING TRAFFIC ENGINEERING IN SOFTWARE DEFINED NETWORKING
}

\author{
Mouhammd Alkasassbeha, ${ }^{\mathrm{a}, *}$, Ghazi Al-Naymat ${ }^{\mathrm{a}}$, Mohammad Alauthman ${ }^{\mathrm{b}}$, Esra \\ Edenat $^{\mathrm{c}}$ \\ ${ }^{a}$ King Hussein Faculty of Computing Sciences Princess Sumaya University for technology, Jordan \\ ${ }^{b}$ Department of Computer Science, Faculty of information technology, Zarqa University, Jordan \\ ${ }^{c}$ Department of Computer Science, Faculty of information technology, mutah University, Jordan
}

\begin{abstract}
The digital society is an outcome of the Internet which has nearly made everything connected and accessible no matter where or when. Nevertheless, despite the fact that conventional IP networks are complicated and very hard to manage, they are still widely adopted. The already established policies make the network configuration/reconfiguration a complex process that reacts to errors, load, and modifications. The prevailing networks are vertically integrated which makes things more and more complicated: Data planes and control are strapped together. Software-defined networking is a model that is meant to solve this issue by splitting the vertical integration and detaching the networks control logic from the implicit routers and switches; this could be achieved by reinforcing centralization of network control and making the network programmable. In this work, we worked to implement MPLS networks with SDN, to enhance the traffic engineering over the network, and to minimize the network delay and latency, with minimum cost using three of the different SDN networks. The experiment results showed the advantage of the proposed approach for reducing the network delay, comparing with previous studies. Where the average of network delay in our approach reaches to 3.01 milliseconds.
\end{abstract}

Keywords: Software-Defined Networking (SDN), Traffic Engineering

\footnotetext{
${ }^{*}$ Corresponding author

Email addresses: m. alkasassbehepsut.edu. jo (Mouhammd Alkasassbeh), g.naymat@psut.edu. jo (Ghazi Al-Naymat), mal authman@zu.edu. jo (Mohammad Alauthman), eedenategmail.com (Esra Edenat)
} 
(TE),Multiprotocol Label Switching (MPLS), Load Balancing, Quality of Service (QoS),Bat Algorithm, Dijkstra.

\section{Introduction}

The allotted control and transport network protocols operating inside the routers and switches are the essential technologies that enable data, in the frame of digital packets, to move around the world. Despite their broad usage, regular IP networks 5 are complicated and difficult to handle [1, 2]. To express the required high-level network policies, network operators demand to configure every single network device independently applying low-level and often vendor-specific commands. In addition to the configuration complexity, network environments have to handle the dynamics of faults and adapt to load changes. Automatic reconfiguration and response devices are virtually missing in existing IP networks. Implementing the needed policies in such a dynamic environment is consequently extremely challenging.

To make it even more difficult, prevailing networks are also vertically integrated. The control plane (that decides how to handle network traffic) and the data plane (that forwards traffic according to the decisions made by the control plane) are strapped inside the networking devices, decreasing flexibility and preventing modification and development of the networking infrastructure. The transition from IPv4 to IPv6, began more than a decade ago and still largely inadequate, bears witness to this challenge, while in fact, IPv6 served merely a protocol update. Due to the passivity of current IP networks, a novel routing protocol can take five to ten years to be completely produced, estimated, and used. Furthermore, a clean-slate plan to change the Internet architecture (e.g., replacing IP) is viewed as a daunting mission not possible in practice [3, 4]. Finally, this situation has increased the capital and operational charges of operating an IP network. Software-defined networking (SDN) is a novel networking paradigm that provides the facility to reduce the constraints of available network infrastructures [5].

\subsection{Software-Defined Networking (SDN)}

As stated before, SDN is a new networking paradigm that promises to reduce the restrictions of current network infrastructures. First, it splits the vertical integration 


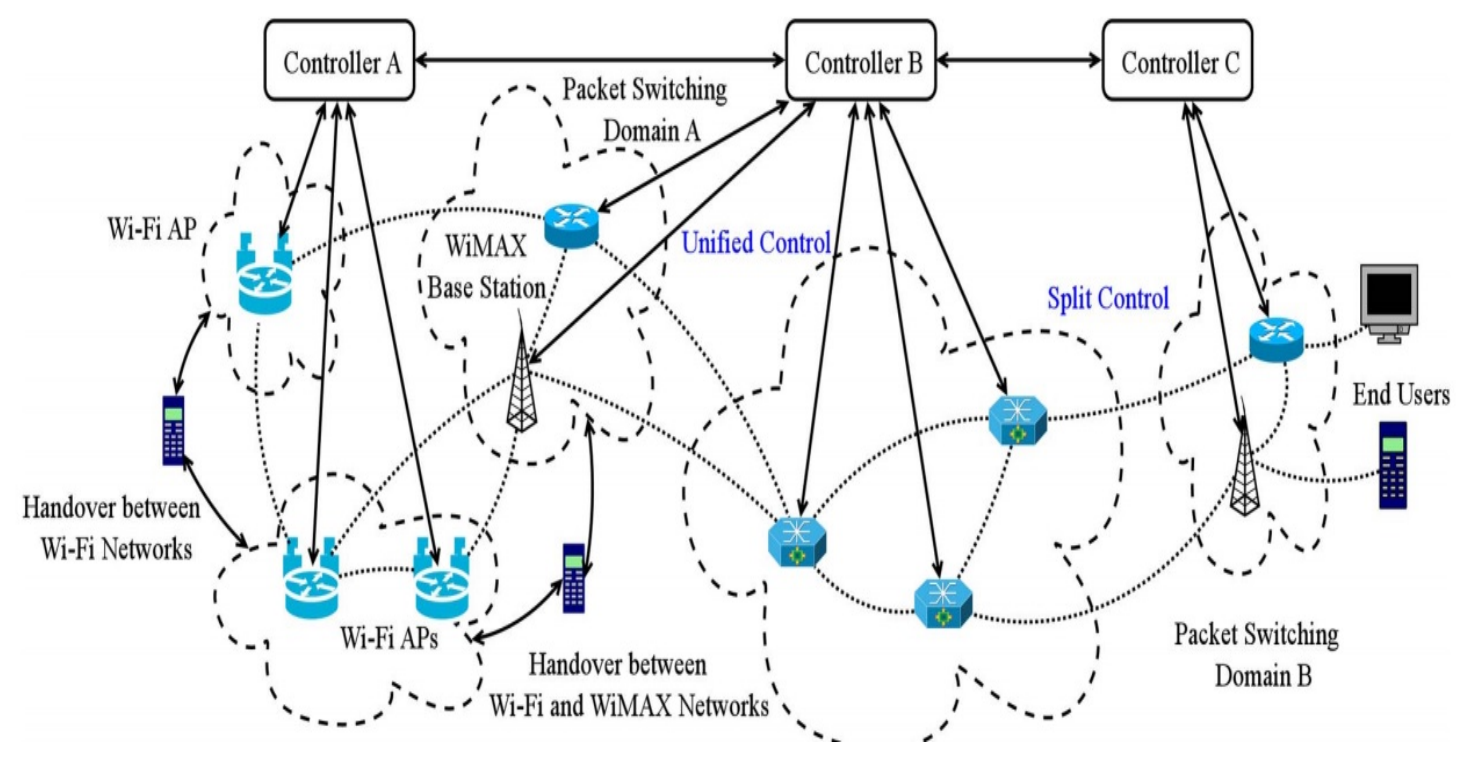

Figure 1: SDN architecture [1]

by detaching the networks control logic from the underlying routers and switches that assist the traffic. Second, by separating the control and data planes, network switches grow simple forwarding devices and the control logic is realized in a logically centralized controller, simplifying policy implementation and network reconfiguration and development [6]. A detailed design of this architecture is presented in Fig. 1] It is necessary to indicate that a logically centralized model does not assume a physically centralized system [7]. In fact, the need to guarantee sufficient levels of performance, scalability, and reliability would hinder such solution. Alternatively, SDN network designs resort to physically distributed control planes [7].

SDN enables a network to be programmed so that its function can be adjusted actively on call and in a fine-grained behavior. It is a unique networking model, where the control plane and the data plane are departed. The idea behind SDN is to simplify network management and allow change, i.e., to promote and use new network applications and services easily, also to run and optimize network performance throughout high-level policy enforcement [8].

The SDN was basically invented to describe the plans and work around OpenFlow 
at Stanford University, USA [9]. As originally defined, SDN indicates to a network between the description of network policies, their use in switching hardware, and the forwarding of traffic. This separation is core to the sought flexibility, splitting the network control problem into tractable parts, and getting it accessible to perform and 
offer new abstractions in networking, simplifying network management and facilitating network development and modification.

\subsection{TRAFFIC ENGINEERING}

A main difficulty with underlying communication network is the dynamic essence of the network applications and their environment. This means that the performance elements of the conveyed data flows, like Quality of Service (QoS), may differ over time. The applications run in a broad span of environments. For the applications to function efficiently, the underlying network should be quite flexible to dynamically change in response to any modifications in the application requirements and their environment. The available programs are either based on static or overprovisioned overlay networks or need the applications to adapt following the network performance [8].

An outstanding action to approach this difficulty is by Traffic Engineering (TE), which is the manner of interpreting the network state, predicting and adjusting the forwarded data load over the network resources. It is a procedure employed to readjust the traffic routing to the settings in the network state. The purpose of TE is to evolve network performance and QoS by effective management of resources, which can decrease operation charge too. The QoS procedures allocate the available resources to the prioritized traffic to bypass congestion for this traffic. However, these procedures do not afford extra resources to the traffic that demands QoS. The conventional routing techniques do not offer any mechanism to allocate network resources optimally [10] [12].

To approach this problem, the research community commenced managing traffic engineering and suggested innovative ideas to promote network robustness in response to the expansion of traffic demands. Traffic engineering decreases the service degradation due to congestion and failure. Moreover, to optimize these heterogeneous networks, both traditional networks and SDN networks, some Traffic Engineering (TE) systems have been proposed. Most are based on tweaking wide range TE and routing mechanism, such as ECMP, IS-IS, and MPLS [8].

In this work, a hybrid approach scenario had been built for using the MPLS to get more professionally network for transmitting a huge amount of traffic. Here in, we fo- 
cused to minimize the network delay and cost for sending packets through the network. EFD. The KLL process observes the TCP buffer and identifies the flows when in a 
given period the buffer passes the designated threshold. This decreases the rules for TE because if each mice-flow is managed by a specific flow rule, it will lead to a bottleneck and restrict the scalability. The benefit of employing group table is that it saves bandwidth as one single group message can refresh a set of flows when the traffic allocation is changed. It applies a weighted routing algorithm which forwards aggregated traffic into various paths by weighing the current network load while calculating the routes [13].

Farhadi et al. [14] present a tag-based classification architecture, where the switches tag the packets based on the application classes. This way the network operator can implement various policies for each of the application classes. The tag is also employed as an identifier for matching the packets which decrease the matching overhead. After a tagged packet is conveyed to the destination edge switch, the switch eliminates the tag and displays the needed actions, if there is any action, and forwards the packet to the destination host. The empirical result confirms that method is $3 \mathrm{~ms}$ faster than the matching applying hash-based approaches like OpenFlow field matching, and degrades processing overhead. To work out the backward compatibility, unlike MPLS, the tag is added to the end of the packet rather than of its middle [14]. This way, if the changeable length packet is confirmed, there is no need for whole packet parsing. Oppositely, the whole packet should be parsed. The downside of this approach is that it needs modifications in the switch internal by adding a new API to the switch data plane.

Zafar et al. [15] examined how to unite application awareness with SDN networks and how to categorize traffics with high accuracy. A framework named Atlas is presented, which is able of organizing the traffic in the network and implementing higher layer policies. The given framework applies a C5.0 ML tool to classify the flows based on the application types. It reveals $94 \%$ accuracy. The Atlas framework can classify every precise application. It can classify every VoIP application specifically rather than classifying them as a common VoIP flows. So framework should be scalable so the application detection and implementing application-aware policy is arranged in a soft and consecutive manner. The deployment of this model needs the users to install software agents on their mobile devices. These brokers gather data such as Netstat logs for 
every application [15]. forwarding table updates. Since QNR applies the SDN facilities for resource reallocation, it is fit for networks with dynamic traffics in which the resource partitioning causes an extraordinary packet loss for high flows. The problem was mathematically 
expressed in the formation of binary linear programming. To work out the corresponding optimization problem, a scheme was introduced. The performance of the suggested scheme was compared with the shortest-path by three metrics 1) SFTC), 2) delay, and 3) packet loss. In all of the test forms, the SFTC and delay increased more than 90 and 60 times, sequentially. Additionally, to completely judge the influence of QNR on network throughput, the packet loss was compared in a simple topology which proved a significant reduction in the packet loss. Eventually, QNR and RQNR were compared concerning time complexity view. In this way, it is noted that the suggested forwarding table procedure (which is used in RQNR) reduces the overhead of FTs update by reducing the number of dynamic flows (the forwarding table rates range between 15\% and $90 \%)$ [18].

Sinha et al. [19] proposed hybrid model where MPLS and SDN models synchronize and harmonize together to allow the stable transition. Particularly, the author employs the regular MPLS data plane concurrently with an uncomplicated and extensible control-plane based on OpenFlow and SDN to develop a hybrid operation model. The traffic is divided using forwarding equivalence classes at the ingress router, which can be updated via a centralized controller using OpenFlow. This model can achieve conflict-free separation of centralized and decentralized controls. Also, it has been confirmed that a centralized controller can provision traffic flows better compared to decentralized based programs using MPLS. Hence, this model presents a better option for the stable transition of a legacy network to a SDN network.

Kitsuwan et al. [20] introduced a model to decrease both the number of constant flow entries and the number of configuration messages from the controller, described as a hybrid permanent flow (HPF) model, in SDN. In this model, switches in the network are broken into different regions. Two MPLS tags (outer and inner) are selected for a packet routing. The outer tag steers a packet from a source switch to an edge switch in its destination region. The inner tag navigates the packet from the edge switch to the destination switch in the local region. A mathematical formulation to define a group of switches in every region so the total number of constant flow entries in the network is reduced. The NSF topology and a sample topology was employed. In the NSF topology, the switches are assorted into three regions. The interpretation of 
the number of flow entries for a sample topology was offered. The performance of the HPF plan was examined. Results from the examination showed that $70 \%$ of the number of constant flow entries and $80 \%$ of the number of configuration messages had decreased in comparison with the traditional schemes. These decreases require a lower bandwidth in the control plane, a lower process of CPU in the controller, and a smaller TCAM memory size in every switch.

Kosugiyama et al. [21] used a heuristic approach to aggregate flows for operating application flows in SDN. The recommended approach sights to reduce the number of flows while every flow contents its permissible delay. It was assessed in some traffic conditions, and simulation outcomes proved that the plan considerably exceeds simple routing by employing the aggregation. The authors formed a flow aggregation problem without link bandwidth. In a real network, though, flows that require more bandwidth and exceed link bandwidth cause packet loss as a result of QoS control. Therefore, the authors assessed the suggested method in a more realistic model acknowledging link bandwidth. The proposed method decreased the number of flows more efficiently in a network with high link density. Still, the method is not very efficient in a network such as a tree model where the route of flows can be uniquely defined. Nevertheless, real networks based on tree models have bypass links for load balancing or decreasing delivery delay.

Amaral et al. [22] SDN TE architecture that reacts to regular scaling issues that occur in SDN optimization based TE systems. The authors noted how an architecture based in a logically hierarchical control plane is befitted for a greater number of scenarios and how TE in such an architecture relies only on the pathfinding algorithm and the way traffic is allotted among paths. Many algorithms for pathfinding and traffic distribution can be utilized in the suggested architecture, and in this work, the results of simulations point out that some network utilization gains can be accomplished using simple shortest paths joined with the use of betweenness centrality for traffic distribution. The use of centrality over shortest paths bounds the gains, and in topologies where there is a small number of similar cost paths, the performance is slightly better than ECMP.

Kurimoto et al.[23] used the multi-layer network design applying MPLS-TP based 
cloud in SDN. The recommended scheme examined in SINET5. This network can diminish the latency between users and improve the TCP throughput. Reliable MPLS-TP paths were located to be switched within $50 \mathrm{msec}$ in a field test. An on-demand controller was employed to be able to cooperate with users through a Web-based interface and cloud services via a REST interface. Additionally, the auto-healing time of a VNF was found to be as short as 30 seconds.

\section{Hybrid Technique for Optimizing Traffic Engineering}

When clients want to send its packets through MPLS network, these packets will arrive at the edge switch with no matching flow rule, the switch asks for detailed information and orders from the controller. The controller's role is to decide on the proper path for this packet and the other matching packets in the network; the controller also generates an equal label and installs it in the switches on the packet's path. This section presents the methodology of our proposed approach, that will be used for the traffic engineer measurement using MPLS in the SDN network. In this paper, we will build a hybrid approach for calculating the needed amount of traffic engineering for each of sender node and receiver node over the network. We used each of Bat and Dijkstra algorithms in our proposed method. Where bat algorithm used for determining the total cost for sending a packet from customer edge to another using different parameters (bandwidth, available capacity, propagation delay, and packet size). While Dijkstra algorithms used to select the appropriate candidate of shortest path, which will be used to transfer data packet between customer's edges in the network (Fig 2). 


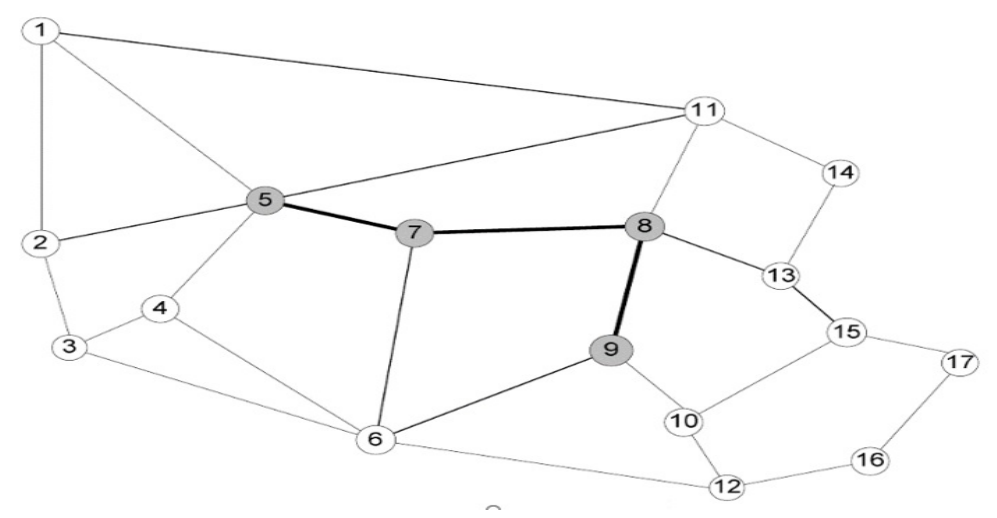

Figure 2: Shortest Path

The main idea of the proposed approach is to find the shortest destination between sender edge of the client, and the receiver edge of another client through the MPLS network. Customer edge (CE) is a special router used only by the client, and connected directly with provider edges (PE) in the SDN network. Where customer edge can use the IP methods only, and can't use the MPLS settings and configuration, while provider edges are working as half IP, and half MPLS. Fig. 3 represents the general framework.

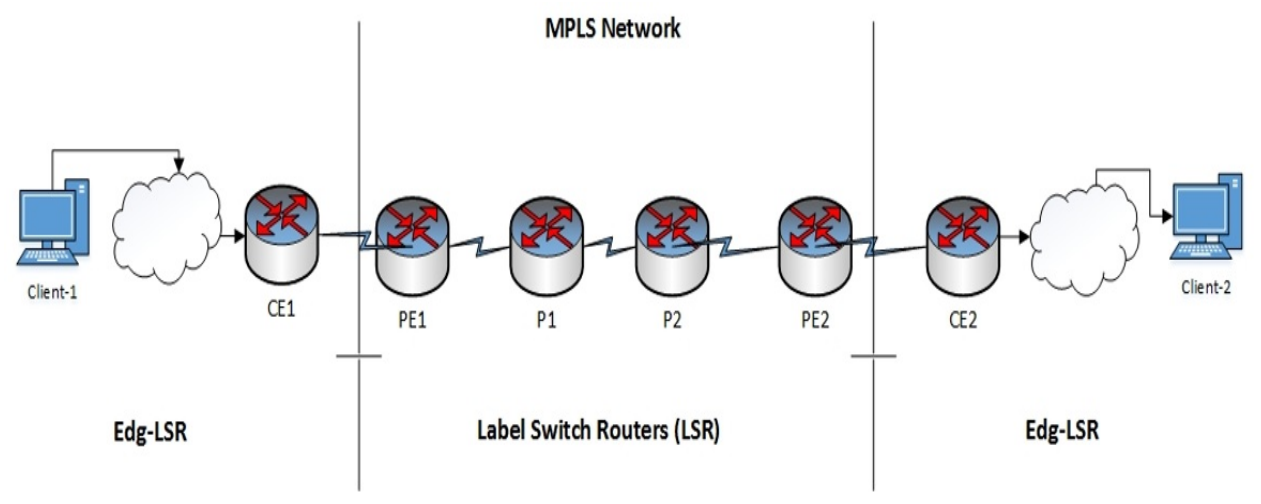

Figure 3: General framework

In this paper, the proposed approach aims to find the Label-Switched Path (LSP) 285 between two customer edges, where LSP describe the destination path for the packets between sender edge (CE1), and the receiver edge (CE2), through the MPLS network. 
Fig. 4 shows the main phases of our research, where the proposed approach includes each of IP model phase, Edge-LSR phase, and intermediate model phase.

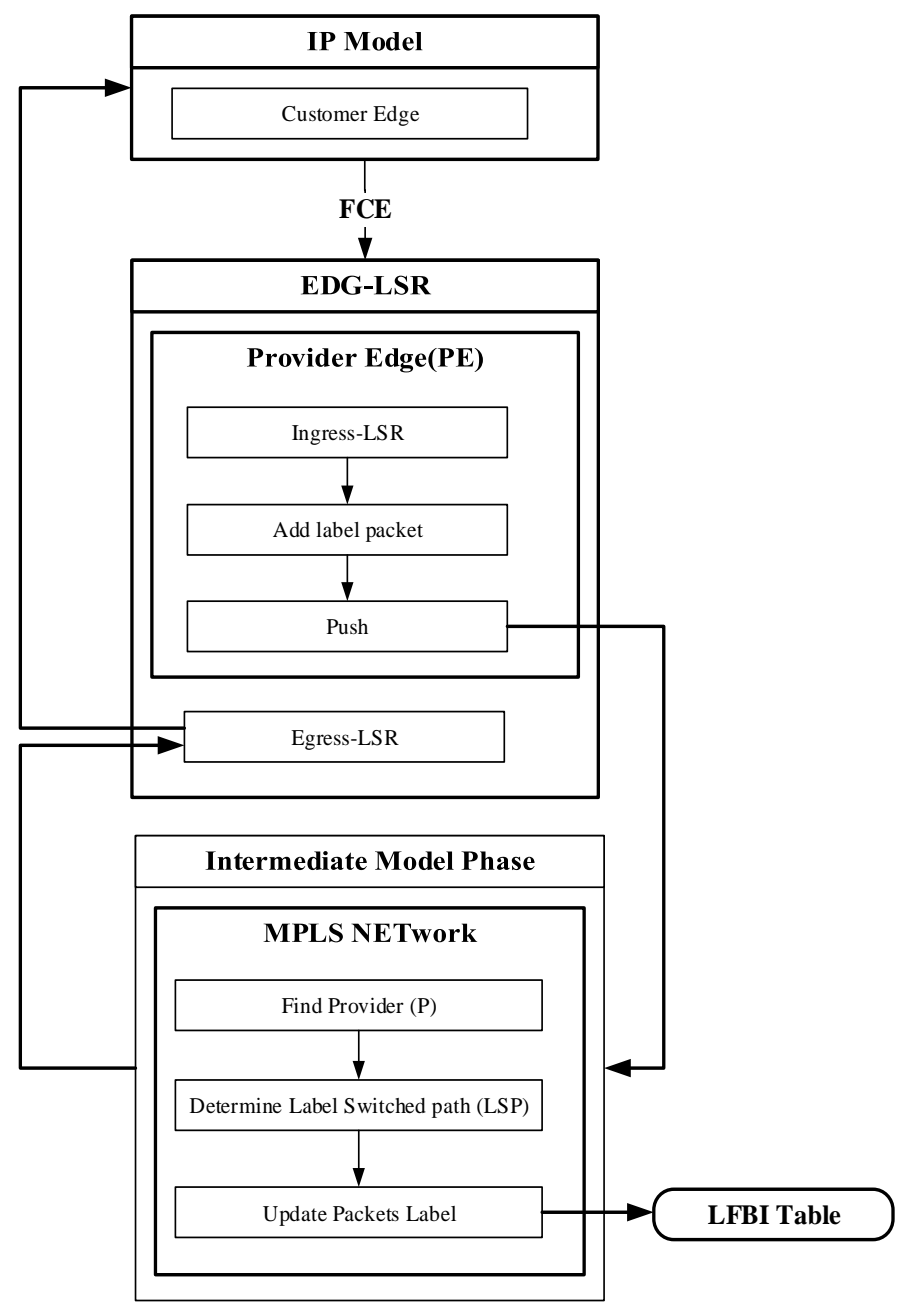

Figure 4: Our Model Phases

\subsection{IP Model}

290

Each router performs an IP lookup (routing), determines a next-hop based on its routing table (label forwarding information base), and forwards the packet to that next- 
hop. Forwarding and pop for every router, each making its own independent routing decisions, until the final destination is reached. In the proposed approach, we will use the IP model phase to connect traditional IP networks with the label switching in MPLS. IP model phase is considered as the both of initial process, and the final process in our proposed approach. The router in this phase uses only the IP mode, and cant use MPLS settings. The initial process is started when the client sends packets through customer edge (CE1), to another client (CE2), the packets are known as Forward Error Correction (FEC), which mean the group of packets from sender router (CE) to the service provider edge' router (PE). While the final process is finished when IP packets reach to the receiver customer edge (CE2).

\subsection{Edge-LSR Phase}

The second phase in the proposed system is the Edge-LSR phase. The routers in this phase receive the IP packets (FEC) from the previous phase using Ingress-LSR routers. Ingress-LSR routers working to receive the IP packets, then adding the label for this packets, and finally forwarding this packets using push label process into a destination for the Intermediate Model phase. After Intermediate Model phase is done, the label packets reach to the Egress-LSR routers, this routers working to remove labels over the packets, and send it to out the MPLS mode into IP routers (customer edges).

\subsection{Intermediate Model Phase}

This is the last phase in the proposed system, and considers as the most important phase, because it's working as the controller over the network, and manage the optimal traffic flow and traffic engineering. The routers in this phase using only MPLS settings (Label Switch Router (LSR), and not connected directly with IP routers (CE), these routers depend on the label only for packet exchange. The proposed approach uses the intermediate model phase to determine label switched path (LSP) in the MPLS, its identify the providers $(\mathrm{P})$ over the destination. In this phase, the label packets will be updated for every router, the values of the updated label will be saved temporarily in the label forwarding information base (LFIB) table. 


$$
A i=C i-B i
$$

Where the $B i$ is current bandwidth load. Then we poll the counters in the SDN switches and calculate the current load $B i$ at the time $T$ using Equation (2):

$$
B i(t)=\frac{(n i(t)-n i(t-T))}{T}
$$

Where $n i(t)$ is the counter value, $T$ is the polling period. According to the formula 
(1) and (2), the available bandwidth on a given path $P$ for each E $i$ will be:

$$
B W p=M I N(a i)
$$

Propagation delay is the time for one bit to propagate from source to destination at propagation speed of the link, Equation (4) calculate the total of propagation time:

$$
\text { Propagationdelay }=F S T+L M D+Q D+N P D
$$

Where FST is the frame serialization time (transmission time), LMD is the link media delay, QD is the queuing delay, and NPD is the node processing delay.

$$
\begin{gathered}
F S T=\frac{S}{R} \\
L M D=\frac{E i}{P d}
\end{gathered}
$$

350

$$
Q D=\frac{Q}{R}
$$

Where $R$ is the link data rate (bits/second), $S$ is the packet size (bits), $P d$ is the processing delay, and the $Q$ is queue depth (bits). To calculate final delay $(f d)$ in the proposed network, we will find the packet processing time (ppt), which it's the time elapsed between the arrival packet in queue, and the time of packet to processed.

$$
f d=p p t 1+t t+t q+t p+p t t 2
$$

where $t t$ is the transmission time (serialization time), $t q$ is the queuing delay, $t p$ is the propagation time, $p p t 1$ is source distance in the shortest path $P$, and ppt2 is the final distance in the same path $P$. According to all of the above Equations, the final cost in the distance path $P$ for the proposed network will be calculated using the proposed formula 9 :

$$
\text { FinalCost }=\frac{(t p * f d)}{B W p}+S
$$


The optimization of traffic engineering in the SDN with MPLS for different path between nodes, can be calculated by the Equation below:

$$
A=\frac{(C * H)}{T}
$$

Where $A$ : traffic density, $C$ : number of calls arrivals during time $T$, and $H$ : average holding time.

\subsection{Bat Algorithm}

Optimization problems are very common to be found in many different applications [28], in the proposed approach, Bat algorithm is used and extended to solve multiobjective optimization problems. The proposed multi-objective bat algorithm (MOBA) is first validated against a subset of test functions [29], and then applied to solve multiobjective design problems, in our approach, it used for selecting shortest distance with minimum cost distance in the SDN network. We are looking for efficiently results for the proposed algorithm using Micro-bats type of bat, which depends on echolocation.

\section{Experiment Results}

We conducted four groups of experiments according to the size of request (200, $300,400,500)$ bytes. Each group of experiments are divided into three blocks according to the number of nodes in the network $(30,50$, and 70) with 65 links in each network. We added new factors for the results, such as total cost, shortest path, network delay, queue delay, and bandwidth. The Table 1 shows the groups of experiments. Moreover, We applied each group of experiments 37 times on the different cases of source nodes.

\subsection{Experiment Example}

According to the EXP 1, the shortest path for sending 200 bytes of data from the source (node 1) to the destination (node 30) will be shown in Figure 5. The Dijkstra's algorithm finds the best shortest path from (node 1) to (node 30)which is $[2,4,10,5$, $3,20,24,19,22]$. Another parameter will be calculated according to bat algorithm is the network latency (final delay)which is equal to 0.564 milliseconds, and the needed 
Table 1: The list of experiment groups

\begin{tabular}{|l|l|l|}
\hline Experiment & Request size (bytes) & Number of Nodes \\
\hline EXP 1 & 200 & $30,50,70$ \\
\hline EXP 2 & 300 & $30,50,70$ \\
\hline EXP 3 & 400 & $30,50,70$ \\
\hline EXP 4 & 500 & $30,50,70$ \\
\hline
\end{tabular}

cost for sending 200 bytes of packets through the network from node to other till the destination is 0.0089 call $/$ second, while the available capacity is $479.32 \mathrm{Mbps}$.

Figure 5 shows how the sending packets through the MPLS network with SDN from the source node (node 1). Then, the packets will move from IP model to the label model, and the network will determine the next node (receive node). In the previous example, node 2 called Ingress-LSR, and it will receive the packets from node 1, node 2 is working with half IP and half label technique. Each node in the shortest path [4, 10, 5, 3, 20, 24, 19] will be working only with Label technique, and they called LSR nodes. While the node 22 is working with half IP technique and half label, it's called Egress-LSR. The For the bat algorithm Figure 6 shows how it ran with iteration equal 50.

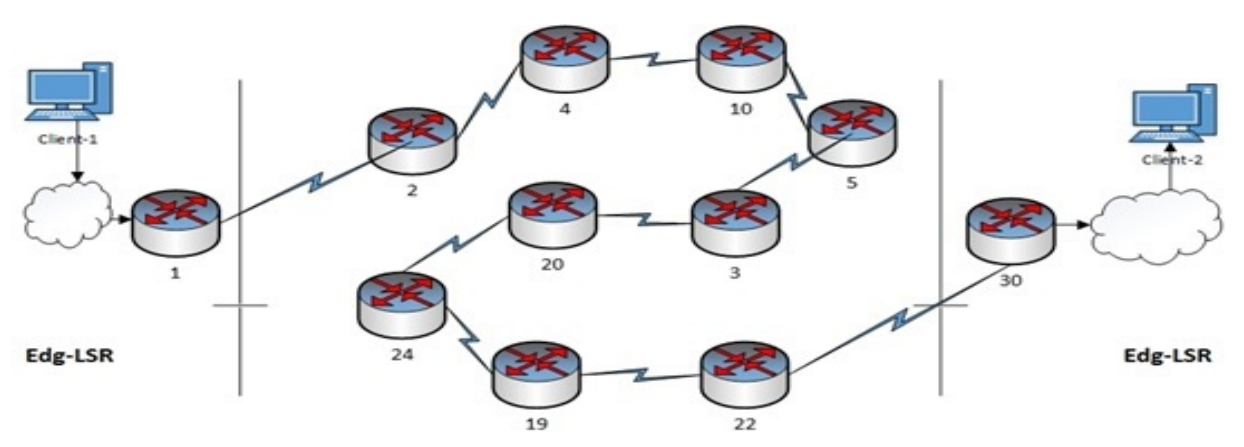

Label Switch Routers (LSR)

Figure 5: An example of Experiment 1 


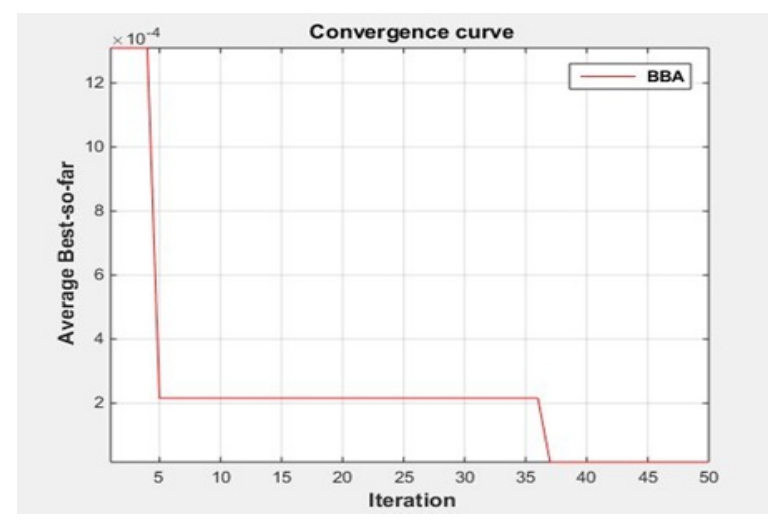

Figure 6: Running Bat Algorithm

\subsection{Results and discussion}

We calculate the average of the traffic density for each experiment according to Equation (10). In the previous example, the traffic density for the selected path is equal 62.06.

\subsubsection{Network Delay}

Network delay; is one of the most important parameters in the proposed approach, we tried to reduce the delay values of the network comparing with previous studies. The Table 2 shows the details for the maximum delay over all links in all experiments:

Figure 7 below shows the total average of maximum delay over all links in four experiments.To compare the final delay in the proposed approach with the previous studies, we will compare that using the same data set for the Agarwal et al. [30] as shown in Figure 8, which contain of 15 node topology, We can notice the difference of two results between the proposed approach with other approaches for each experiments, which consider as the positive factor for the proposed approach. 
Table 2: Network Delay

\begin{tabular}{|c|c|c|}
\hline Experiments & Number of Nodes & Average Delay \\
\hline \multirow[t]{4}{*}{ EXP 1} & 30 & 0.693015072 \\
\hline & 50 & 0.695972389 \\
\hline & 70 & 0.756549509 \\
\hline & Average & 0.71517899 \\
\hline \multirow[t]{4}{*}{ EXP 2} & 30 & 0.791981026 \\
\hline & 50 & 0.941954024 \\
\hline & 70 & 1.144160616 \\
\hline & Average & 0.959365222 \\
\hline \multirow[t]{4}{*}{ EXP 3} & 30 & 1.155042036 \\
\hline & 50 & 1.028360054 \\
\hline & 70 & 1.04885195 \\
\hline & Average & 1.077418013 \\
\hline \multirow[t]{4}{*}{ EXP 4} & 30 & 1.294034558 \\
\hline & 50 & 1.249904775 \\
\hline & 70 & 1.246094201 \\
\hline & Average & 1.263344511 \\
\hline
\end{tabular}

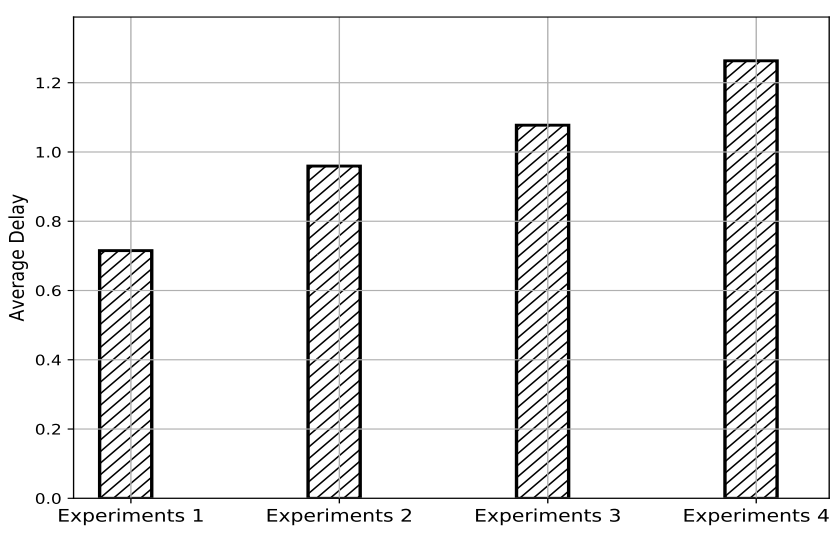

Figure 7: Experiments Average Delay 


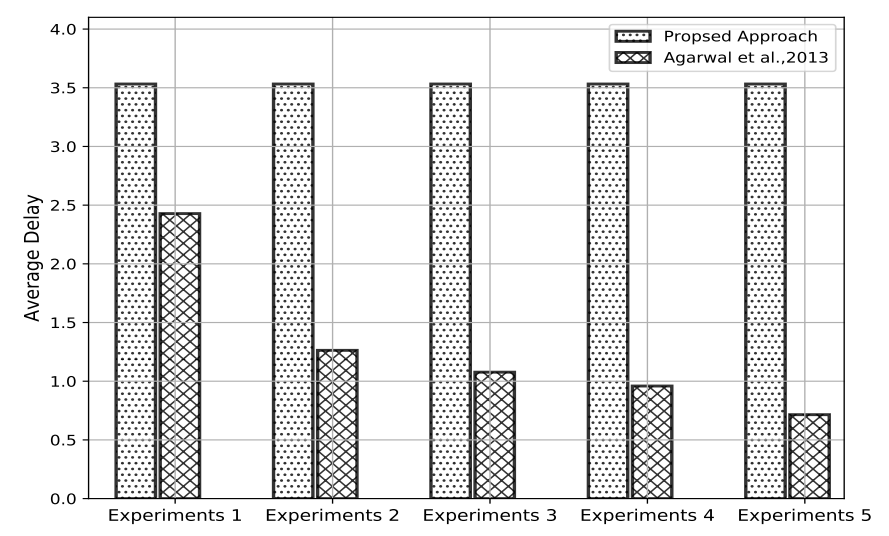

Figure 8: Comparison Average Delay Over All Links (All Experiments)

\subsubsection{Traffic Density}

For the Traffic density, the experiment results show the traffic density for all experiments, Figure 9 below shows the total traffic density for the four experiments.

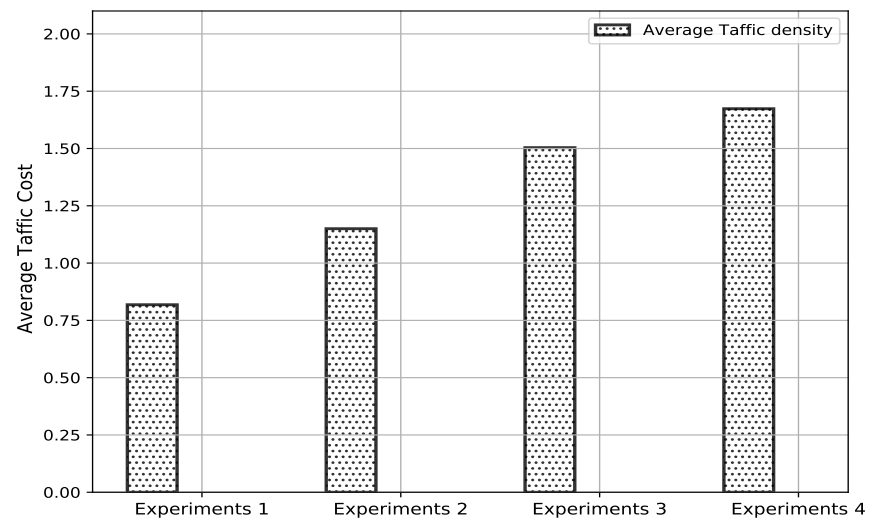

Figure 9: Experiments Traffic Density (bps)

We can notify the increasing value of traffic density with maximum packets, which mean the flexibility of the proposed approach for each of big networks and the huge 
packets. The average of density cost for all experiments is $70.03 \mathrm{bps}$.

\subsubsection{Traffic Cost}

Table 3 shows the details of the traffic costs for all experiments:

\begin{tabular}{|l|l|l|}
\hline \multirow{2}{*}{ Experiments } & Table 3: Traffic Cost \\
\hline \multirow{3}{*}{ EXP 1 } & 30 & 0.272733464 \\
\cline { 2 - 3 } & 50 & 0.238619452 \\
\cline { 2 - 3 } & 70 & 0.306838191 \\
\hline \multirow{3}{*}{ EXP 2 } & 30 & 0.301865492 \\
\cline { 2 - 3 } & 50 & 0.327923355 \\
\cline { 2 - 3 } & 70 & 0.52017084 \\
\hline \multirow{3}{*}{ EXP 3 } & 30 & 0.635195344 \\
\cline { 2 - 3 } & 50 & 0.479264822 \\
\cline { 2 - 3 } & 70 & 0.389239157 \\
\hline \multirow{3}{*}{ EXP 4 } & 30 & 0.424216318 \\
\cline { 2 - 3 } & 50 & 0.62814365 \\
\cline { 2 - 3 } & 70 & 0.621184828 \\
\hline
\end{tabular}

We also can notify the increasing value of traffic cost with the maximum packets, and the positive relationship between traffic cost and traffic density. Where the average of traffic cost for all experiments is $1.286 \mathrm{Mbps}$. This positive relationship between average cost and traffic density is reinforced our methodology for selecting the best shortest path with minimum cost, and show how traffic engineering affected by a number of nodes between source nodes and distance nodes. Finally, the increasing of packet size between nodes in the MPLS need more cost and traffic density. However, this increasing provides a higher value of the bandwidth in the network, as it shown in the Figure 10 below: 


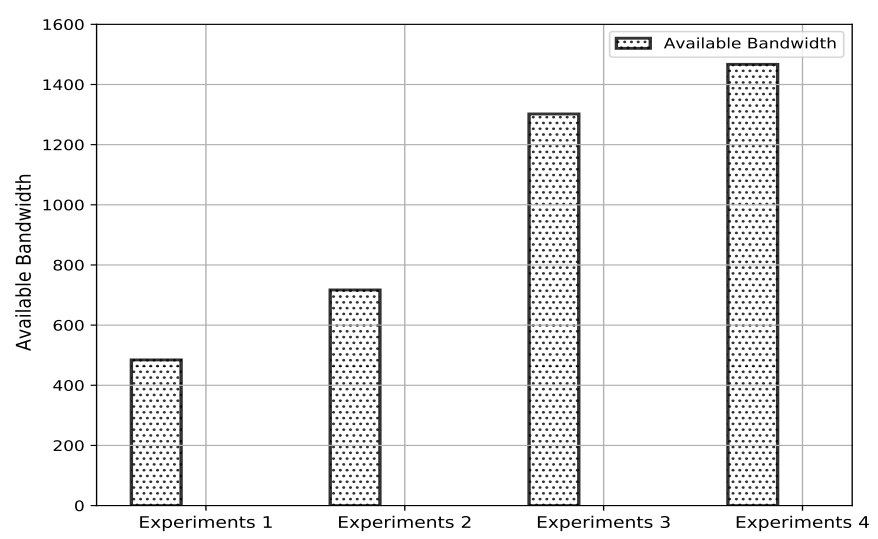

Figure 10: Experiments Available Bandwidth

\section{Conclusion}

Finally, we built a new scenario that considered as a hybrid approach for using the MPLS to get more effectively network for transmitting a huge traffic amount in Software-defined networking. In our approach, we focused to minimize each of network delay and the needed cost for sending packets through the network, and to calculate the maximum value of traffic density over the network. The proposed scenario is aimed to find the shortest path for the destination between the sender node and a receiver node, we used the Dijkstras algorithm to find the best path (shortest) between a list of candidate paths. In the other hand, we used bat algorithm to find each of the needed cost for sending packets with the best traffic density, with minimum network delay.

We conducted four experiments according to the size of requests between nodes (200, 300, 400, and 500 bytes), where each experiment was run on three different networks, 30 nodes, 50 nodes and 70 nodes respectively. The experiment results show the advantage of the proposed approach for reducing the network delay, comparing with Agarwal et al.[30] study. Where the average of network delay in their study reach to 3.532 milliseconds, while in our approach, the average of network delay reaches to 3.01 
milliseconds. The results show the sending of packet size between nodes costs more that of the traffic density when the increases of packet size. However, the increasing of traffic density and cost are providing a higher value of the bandwidth in the network.

[1] W. Xia, Y. Wen, C. H. Foh, D. Niyato, H. Xie, A survey on software-defined networking, IEEE Communications Surveys \& Tutorials 17 (1) (2015) 27-51.

[2] Q. Yan, F. R. Yu, Q. Gong, J. Li, Software-defined networking (sdn) and distributed denial of service (ddos) attacks in cloud computing environments: A survey, some research issues, and challenges, IEEE Communications Surveys \& Tutorials 18 (1) (2016) 602-622.

[3] H. Farhady, H. Lee, A. Nakao, Software-defined networking: A survey, Computer Networks 81 (2015) 79-95.

[4] Z. Qin, G. Denker, C. Giannelli, P. Bellavista, N. Venkatasubramanian, A software defined networking architecture for the internet-of-things, in: Network Operations and Management Symposium (NOMS), 2014 IEEE, IEEE, 2014, pp. $1-9$.

[5] K. Kirkpatrick, Software-defined networking, Communications of the ACM 56 (9) (2013) 16-19.

[6] H. Kim, N. Feamster, Improving network management with software defined networking, IEEE Communications Magazine 51 (2) (2013) 114-119.

[7] S. Jain, A. Kumar, S. Mandal, J. Ong, L. Poutievski, A. Singh, S. Venkata, J. Wanderer, J. Zhou, M. Zhu, et al., B4: Experience with a globally-deployed software defined wan, in: ACM SIGCOMM Computer Communication Review, Vol. 43, ACM, 2013, pp. 3-14.

[8] M. R. Abbasi, A. Guleria, M. S. Devi, Traffic engineering in software defined networks: A survey, Journal of Telecommunications and Information Technology (4) (2016) 3 . 
[9] D. Kreutz, F. M. Ramos, P. E. Verissimo, C. E. Rothenberg, S. Azodolmolky, S. Uhlig, Software-defined networking: A comprehensive survey, Proceedings of the IEEE 103 (1) (2015) 14-76.

[10] I. F. Akyildiz, A. Lee, P. Wang, M. Luo, W. Chou, A roadmap for traffic engineering in sdn-openflow networks, Computer Networks 71 (2014) 1-30.

[11] R. Muñoz, R. Vilalta, R. Casellas, R. Martínez, F. Francois, M. Channegowda, A. Hammad, S. Peng, R. Nejabati, D. Simeonidou, et al., Transport network orchestration for end-to-end multilayer provisioning across heterogeneous sdn/openflow and gmpls/pce control domains, Journal of Lightwave Technology 33 (8) (2015) 1540-1548.

[12] G. Al-Naymat, M. Al-Kasassbeh, N. Abu-Samhadanh, S. Sakr, Classification of voip and non-voip traffic using machine learning approaches., Journal of Theoretical \& Applied Information Technology.

[13] R. Trestian, G.-M. Muntean, K. Katrinis, Micetrap: Scalable traffic engineering of datacenter mice flows using openflow, in: Integrated Network Management (IM 2013), 2013 IFIP/IEEE International Symposium on, IEEE, 2013, pp. 904907.

[14] H. Farhadi, A. Nakao, Rethinking flow classification in sdn, in: Cloud Engineering (IC2E), 2014 IEEE International Conference on, IEEE, 2014, pp. 598-603.

[15] Z. A. Qazi, J. Lee, T. Jin, G. Bellala, M. Arndt, G. Noubir, Application-awareness in sdn, in: ACM SIGCOMM computer communication review, Vol. 43, ACM, 2013, pp. 487-488.

[16] K. T. Dinh, S. Kukliński, W. Kujawa, M. Ulaski, Msdn-te: Multipath based traffic engineering for sdn, in: Asian Conference on Intelligent Information and Database Systems, Springer, 2016, pp. 630-639.

[17] P. Thorat, S. Jeon, H. Choo, Enhanced local detouring mechanisms for rapid and lightweight failure recovery in openflow networks, Computer Communications 108 (2017) 78-93. 
[18] M. M. Tajiki, B. Akbari, N. Mokari, Optimal qos-aware network reconfiguration in software defined cloud data centers, Computer Networks 120 (2017) 71-86.

[19] Y. Sinha, S. Bhatia, V. S. Shekhawat, G. Chalapathi, Mpls based hybridization in sdn, in: Software Defined Systems (SDS), 2017 Fourth International Conference on, IEEE, 2017, pp. 156-161.

[20] N. Kitsuwan, S. Ba, E. Oki, T. Kurimoto, S. Urushidani, Flows reduction scheme using two mpls tags in software-defined network, IEEE Access 5 (2017) 1462614637.

[21] T. Kosugiyama, K. Tanabe, H. Nakayama, T. Hayashi, K. Yamaoka, A flow aggregation method based on end-to-end delay in sdn, in: Communications (ICC), 2017 IEEE International Conference on, IEEE, 2017, pp. 1-6.

[22] P. Amaral, P. F. Pinto, L. Bernardo, F. Silva, Sdn based traffic engineering without optimization: A centrality based approach, in: Communications (ICC), 2017 IEEE International Conference on, IEEE, 2017, pp. 1-7.

[23] T. Kurimoto, S. Urushidani, H. Yamada, K. Yamanaka, M. Nakamura, S. Abe, K. Fukuda, M. Koibuchi, H. Takakura, S. Yamada, et al., Sinet5: A low-latency and high-bandwidth backbone network for $\mathrm{sdn} / \mathrm{nfv}$ era, in: Communications (ICC), 2017 IEEE International Conference on, IEEE, 2017, pp. 1-7.

[24] S. Kadry, A. Abdallah, C. Joumaa, On the optimization of dijkstras algorithm, in: Informatics in Control, Automation and Robotics, Springer, 2011, pp. 393-397.

[25] S. Sanan, L. Jain, B. Kappor, Shortest path algorithm, International Journal of Application or Innovation in Engineering \& Management (IJAIEM) 2 (7) (2013) $316-320$.

[26] H. Wang, Y. Yu, Q. Yuan, Application of dijkstra algorithm in robot pathplanning, in: Mechanic Automation and Control Engineering (MACE), 2011 Second International Conference on, IEEE, 2011, pp. 1067-1069. 
525

[29] X.-S. Yang, X. He, Bat algorithm: literature review and applications, International Journal of Bio-Inspired Computation 5 (3) (2013) 141-149.

[30] S. Agarwal, M. Kodialam, T. Lakshman, Traffic engineering in software defined networks, in: INFOCOM, 2013 Proceedings IEEE, IEEE, 2013, pp. 2211-2219. 